\title{
Effects of Reproductive Factors on Lauren Intestinal-Type Gastric Cancers in Females: A Multicenter Retrospective Study in South Korea
}

\author{
Yoon Ju Jung1, Hee Jin Kim², Cho Hyun Park ${ }^{3}$, Seun Ja Park', and Nayoung Kim, \\ ${ }^{1}$ Division of Gastrointestinal Surgery, Department of Surgery, Incheon St. Mary's Hospital, College of Medicine, The Catholic University \\ of Korea, Incheon, ${ }^{2}$ Department of Internal Medicine, Gyeongsang National University Changwon Hospital, Gyeongsang National \\ University College of Medicine, Changwon, ${ }^{3}$ Division of Gastrointestinal Surgery, Department of Surgery, Seoul St. Mary's Hospital, \\ College of Medicine, The Catholic University of Korea, Seoul, ${ }^{4}$ Department of Internal Medicine, Kosin University College of Medicine, \\ Busan, ${ }^{5}$ Department of Internal Medicine, Seoul National University Bundang Hospital, Seongnam, and ${ }^{6}$ Department of Internal \\ Medicine and Liver Research Institute, Seoul National University College of Medicine, Seoul, Korea
}

Article Info

Received July 1, 2021

Revised October 5, 2021

Accepted October 21, 2021

Published online January 7, 2022

\section{Corresponding Author}

Nayoung Kim

ORCID https://orcid.org/0000-0002-9397-0406

E-mail nakim49@snu.ac.kr

Seun Ja Park

ORCID https://orcid.org/0000-0003-3217-5115

E-mail parksj6406@daum.net

Yoon Ju Jung and Hee Jin Kim contributed equally to this work as first authors.
Background/Aims: Gastric cancers (GCs), particularly the Lauren intestinal type, show a male predominance. The aim of this study was to investigate the effects of reproductive factors on GCs in females, according to Lauren classification.

Methods: Medical records of 1,849 males and 424 females who underwent radical gastrectomy or endoscopic resection for GCs between 2010 and 2018 were reviewed. The incidences of intestinal-type GCs were compared between males and groups of females stratified according to postmenopausal period. Associations between reproductive factors in females and intestinaltype GCs were analyzed using multivariate models.

Results: The proportions of intestinal-type GCs were significantly lower in premenopausal $(19 \%)$, less than 10 years postmenopausal $(30.4 \%)$, and 10 to 19 years postmenopausal females $(44.1 \%)$ than in males $(61.0 \%)$ ( $p<0.05$ for all). Females $\geq 20$ years postmenopause had a proportion of intestinal-type GCs similar to that in males ( $60.6 \%$ vs $61.0 \% ; p=0.948)$. Multivariate analysis revealed that age (odds ratio [OR], 1.075; 95\% confidence interval [Cl], 1.039 to 1.113; $p<0.001)$ and parity $\geq 3(\mathrm{OR}, 1.775 ; 95 \% \mathrm{Cl}, 1.012$ to $3.114 ; \mathrm{p}=0.045)$ were positively associated with an increased risk of intestinal-type GCs in postmenopausal females, while long fertility duration $(\mathrm{OR}, 1.147 ; 95 \% \mathrm{Cl}, 1.043$ to $1.261 ; \mathrm{p}=0.005)$ was positively associated with an increased risk of intestinal-type GCs in premenopausal females.

Conclusions: There were no significant differences in the proportions of intestinal-type GCs between males and females $\geq 20$ years postmenopause, suggesting that female reproductive factors play a role in the prevention of intestinal-type GC. (Gut Liver 2022;16:706-715)

Key Words: Gastric; Cancer; Female; Reproductive

\section{INTRODUCTION}

The incidence of gastric cancer (GC) is 2-fold to 3-fold greater in males than in females in most populations. ${ }^{1} \mathrm{~A}$ lower GC incidence in females may partly be caused by lower exposure to risk factors, such as cigarette smoking, ${ }^{2}$ alcohol use, Helicobacter pylori infections, ${ }^{3}$ and obesity; however, these factors do not fully explain the disparity. This has led to the hypothesis that sex hormones are in- volved in GC development. This notion is also supported by the observation that the male-to-female ratio in GC incidence rates peaks at 2.5 at the age of 60 years; it then declines to 1.5 , suggesting diminished postmenopausal protection. ${ }^{4}$

Estrogens are involved in various physiologic processes, primarily via binding to estrogen receptors, which are potent transcriptional regulators. Estrogen has oncogenic and angiogenic effects; ${ }^{5}$ it has crucial roles in the cardio- 
vascular, nervous, and immune systems. ${ }^{6}$ Epidemiological studies have demonstrated some level of protection against GC with longer fertility duration and hormone replacement therapy. ${ }^{7}$ Recently, a large cohort study of 333,919 European females demonstrated a negative association of the age at first pregnancy, and a positive association of bilateral ovariectomies, with the incidences of gastric noncardiac cancers. ${ }^{8}$ However, most recent evidence comes from Western studies, and no studies have demonstrated associations of estrogen exposure factors with GC on the basis of histologic subtypes.

GC can be classified into two histologic subtypes according to the Lauren classification (i.e., intestinal and diffuse); these subtypes are distinct in terms of microscopic and gross morphologies, epidemiology, pathogenesis, genetics, and prognosis. ${ }^{9,10}$ Diffuse-type GCs occur more frequently in females and at younger ages; they have a greater tendency to invade the gastric walls and metastasize, leading to a worse prognosis compared with intestinal-type GCs. ${ }^{11}$ In contrast, intestinal-type GCs occur more frequently in males and at older ages; environmental factors, rather than genetic factors, contribute to their development. ${ }^{11}$ Typically, intestinal-type GCs arise through a multi-step process that involves atrophic gastritis, intestinal metaplasia, and dysplasia; these steps are associated with chronic inflammatory processes caused by $H$. pylori. ${ }^{12}$

Sex is a biological status based on reproductive systems and functions, assigned on the basis of chromosomal type.
In contrast, gender includes manners, feelings, and behaviors that are associated with sex stereotypes in a particular culture. ${ }^{13,14}$ Sex is a crucial factor in the pathogenesis, risk, progression, and prognosis of various diseases; it occasionally determines drug responses. ${ }^{15,16}$ However, GC was not much evaluated in terms of sex-specific medicine. We hypothesized that the effects of estrogen on GC development differed on the basis of the Lauren histologic type and reproductive factors. This study aimed to investigate associations between reproductive factors and GC development according to the Lauren classification in a nationwide multicenter study of Korean females.

\section{MATERIALS AND METHODS}

\section{Study populations}

This study included patients who were diagnosed with GCs and underwent endoscopic or curative surgical resections at the St. Mary's Hospital (Seoul), Kosin University Hospital (Busan), and Seoul National University Bundang Hospital (Seongnam), between February 1, 2010, and December 31, 2018. Medical records were retrospectively reviewed. All included patients had been diagnosed with gastric adenocarcinomas or poorly cohesive carcinomas on the final pathology reports of surgical or endoscopic resection specimens. Patients were excluded if they had other non-gastric malignancies within 5 years from the time of

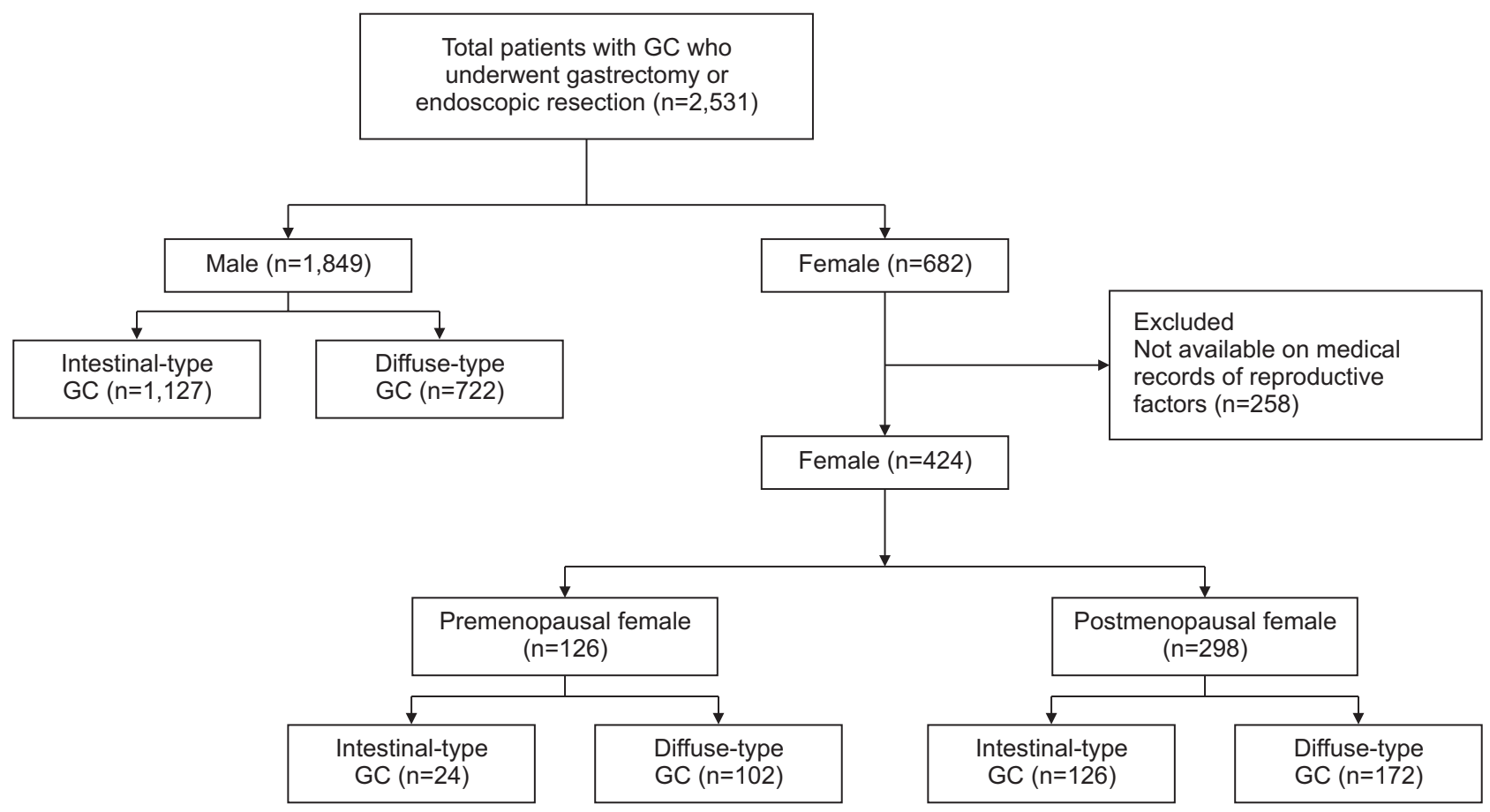

Fig. 1. Algorithm for the inclusion and classification of study participants.

$\mathrm{GC}$, gastric cancer. 
the enrollment and/or if they had incomplete medical records regarding estrogen exposure factors.

The following baseline characteristics were assessed: age, sex, smoking and drinking status, first-degree family history of GC, and body mass index. Clinicopathological data, including final pathology reports and computed tomography results, were collected using the electronic medical chart system. GCs were staged using the 7th edition of the TNM staging system of the American Joint Committee on Cancer (2010), based on the final pathology reports. The location, size, and number of the tumors were evaluated, as were the histologic type and Lauren classification. A patient was considered positive for $H$. pylori infection if at least one of the following yielded positive results: Campylobacter-like organism test and histological examination (hematoxylin and eosin or modified Giemsa staining).

A questionnaire was used to obtain histories regarding reproductive factors, such as the age at first menstrual period, menopausal status, age at last menstrual period, number of live births, use and duration of oral contraceptive pills and/or intrauterine devices, hormone replacement therapy (age at initiation and the total number of years used), history of hysterectomy or oophorectomy, and duration of breast feeding for each pregnancy. Fertility duration was calculated as the interval between the age at menarche and age at menopause in postmenopausal females; it was calculated as the interval between the age at menarche and GC diagnosis in premenopausal females. The study protocol was approved by the Ethical Committees at St. Mary's Hospital (IRB number: KC19RIDE0906), Kosin University Hospital (IRB number: 2020-06-032), and Seoul National University Bundang Hospital (IRB number: B-2002-595104). This study is a retrospective study using medical record review and so informed consent was waived.

\section{Statistical analysis}

SPSS Statistics software (version 22.0; IBM Corp., Armonk, NY, USA) was used to perform the statistical analysis. The chi-square test and the Fisher exact test were used to evaluate associations among categorical variables. The Student t-test was used to evaluate associations among continuous variables. Multivariate logistic regression anal-

Table 1. Clinicopathologic Characteristics of Total Patients According to Sex

\begin{tabular}{|c|c|c|c|}
\hline Characteristics & Male $(n=1,849)$ & Female $(n=424)$ & p-value \\
\hline Age, yr & $61.61 \pm 11.24$ & $57.18 \pm 11.94$ & $<0.001 *$ \\
\hline$<40$ & $48(2.6)$ & $35(8.3)$ & $<0.001^{*}$ \\
\hline $40-49$ & $217(11.7)$ & $76(17.9)$ & \\
\hline $50-59$ & $500(27.0)$ & $121(28.5)$ & \\
\hline $60-69$ & $583(31.5)$ & $121(28.5)$ & \\
\hline$\geq 70$ & $501(27.1)$ & $71(16.7)$ & \\
\hline \multicolumn{4}{|l|}{ Smoking } \\
\hline Never & $760(41.1)$ & $367(86.6)$ & $<0.001^{*}$ \\
\hline Ex/current smoker & $1,089(58.9)$ & $57(13.4)$ & \\
\hline \multicolumn{4}{|l|}{ Drinking } \\
\hline No & $178(9.6)$ & 272 (64.2) & $<0.001^{*}$ \\
\hline Yes & $1,671(90.4)$ & 152 (35.8) & \\
\hline Body mass index, $\mathrm{kg} / \mathrm{m}^{2+}$ & $23.78 \pm 3.09$ & $22.40 \pm 3.23$ & $<0.001^{*}$ \\
\hline$<25$ & $1,224(66.2)$ & $305(79.2)$ & $<0.001^{*}$ \\
\hline$\geq 25$ & 625 (33.8) & $80(20.8)$ & \\
\hline \multicolumn{4}{|c|}{ Helicobacter pylori infection $^{\dagger}$} \\
\hline Negative & $105(48.2)$ & $87(31.0)$ & $<0.001 *$ \\
\hline Positive & 113 (51.8) & $194(69.0)$ & \\
\hline \multicolumn{4}{|l|}{ Tumor location $^{+}$} \\
\hline Lower & 972 (53.6) & $185(44.0)$ & $<0.001^{*}$ \\
\hline Middle & $552(30.5)$ & $180(42.9)$ & \\
\hline Upper & $288(15.9)$ & 55 (13.1) & \\
\hline \multicolumn{4}{|l|}{ Tumor number $^{+}$} \\
\hline Single & 1,079 (92.5) & 408 (97.1) & $<0.001 *$ \\
\hline Multiple & $138(7.5)$ & $12(2.9)$ & \\
\hline Tumor size, $\mathrm{cm}^{\dagger}$ & $4.54 \pm 5.20$ & $4.30 \pm 5.63$ & 0.398 \\
\hline \multicolumn{4}{|l|}{ Lauren classification } \\
\hline Intestinal & $1,127(61.0)$ & $150(35.4)$ & $<0.001 *$ \\
\hline Diffuse & 722 (39.0) & $274(64.6)$ & \\
\hline
\end{tabular}

Data are presented as the mean \pm SD or number (\%).

*Statistically significant, $p<0.05 ;{ }^{\dagger}$ Some data were missing. 
ysis was performed to evaluate risk factors for GC development. All results with $\mathrm{p}<0.05$ were considered statistically significant.

\section{RESULTS}

\section{Baseline clinicopathological characteristics}

The algorithm for participant inclusion and exclusion is shown in Fig. 1. We enrolled 2,531 patients (1,849 males and 682 females) in this study. Among them, 258 females who had incomplete medical records regarding reproductive factors were excluded, and the remaining 424 females were analyzed.

The clinicopathological characteristics of the participants are summarized in Table 1. The mean ages of males and females were $61.61 \pm 11.24$ years and $57.18 \pm 11.94$ years, respectively $(\mathrm{p}<0.001)$. Significantly greater proportions of males were ex-smoker or current smoker (males 58.9\% vs females $13.4 \%$; $\mathrm{p}<0.001)$, used alcohol ( $90.4 \%$ vs $35.8 \%$; $\mathrm{p}<0.001$ ), were obese (body mass index $\geq 25 \mathrm{~kg} / \mathrm{m}^{2}: 33.8 \%$ vs $20.8 \%$; $\mathrm{p}<0.001)$, had GCs in the lower third of the stomach $(53.6 \%$ vs $44.0 \%$; $<<0.001)$, had multiple GCs $(7.5 \%$ vs $2.9 \%$; $<<0.001)$, and had intestinal-type GCs $(61.0 \%$ vs $35.4 \%$; $\mathrm{p}<0.001$ ), compared with females. Compared with males, a significantly greater proportion of females had a positive $H$. pylori infection status (males $51.8 \%$ vs females 69.0\%; $\mathrm{p}<0.001$ ), although the $H$. pylori infection status of many patients was unknown. No significant differences were observed in tumor sizes.

To analyze differences between sexes, we compared the proportions of Lauren subtypes in subgroups classified on the basis of smoking status (never, ex-smoker, or current smoker), drinking status (none, social drinking, or heavy drinking), obesity (body mass index $<25 \mathrm{~kg} / \mathrm{m}^{2}$ or $\geq 25 \mathrm{~kg} / \mathrm{m}^{2}$ ), and $H$. pylori infection (negative or positive). Intestinal-type GCs were prevalent in males, while diffuse-type GCs were prevalent in females in all subgroups (Supplementary Table $1)$.

\section{Proportions of intestinal-type GCs in males and females}

Intestinal-type GCs were significantly less frequent in premenopausal females $(19.0 \%, \mathrm{p}<0.001)$ and postmenopausal females with $<10$ years $(30.4 \%, \mathrm{p}<0.001)$ or 10 to 19 years $(44.1 \%, \mathrm{p}=0.001)$ since menopause, compared with males (61.0\%) (Fig. 2). There was no significant difference in the proportion of intestinal-type GCs between males and $\geq 20$ years postmenopausal females $(60.6 \%$ vs $61.0 \%$; $\mathrm{p}=0.518$ ) (Fig. 2).

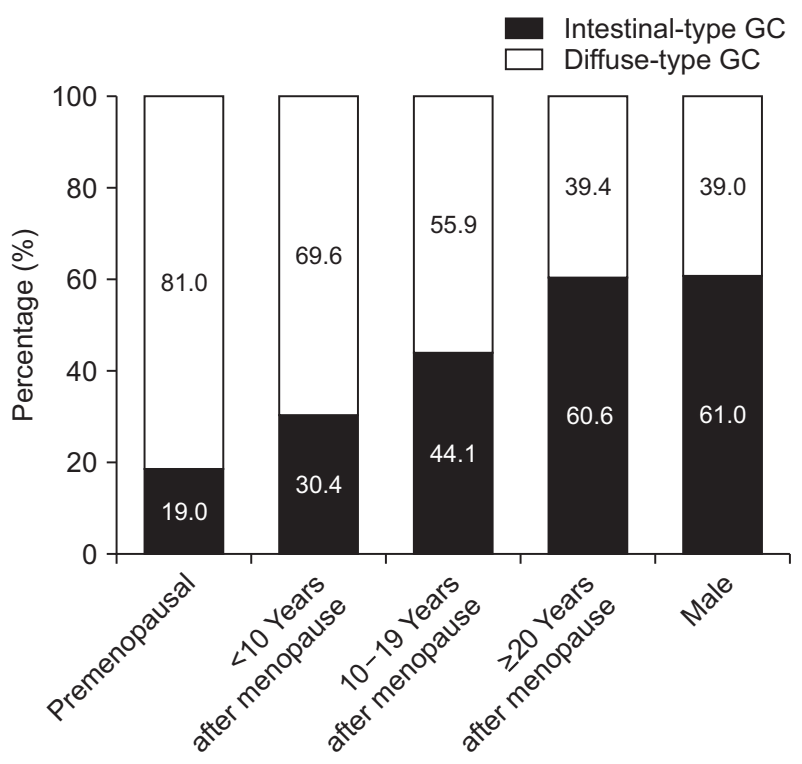

Fig. 2. Proportions of intestinal-type gastric cancers (GCs) in premenopausal and postmenopausal females, compared with in males. Intestinal-type GCs were significantly less frequent in premenopausal $(19.0 \%)$, < 10 years postmenopausal $(30.4 \%)$, and 10 to 19 years postmenopausal females $(44.1 \%)$ than in males $(61 \%)$ ( $p<0.05$ for all). No significant differences in the proportion of intestinal-type GCs were found between males and $\geq 20$ years postmenopausal females $160.6 \%$ vs $61 \% ; p=0.518)$.

\section{Risk factors for intestinal-type GCs in females}

Reproductive factors were compared between intestinaltype and diffuse-type GCs in females (Table 2). Patients with intestinal-type GCs were significantly older than patients with diffuse-type GCs (mean age: $63.02 \pm 10.33$ years vs $53.98 \pm 11.55$ years; $p<0.001)$. Patients with intestinaltype GCs had delayed menarche (intestinal-type GCs of $15.81 \pm 1.89$ years vs diffuse-type GCs of $15.25 \pm 1.80$ years; $\mathrm{p}=0.003)$, greater fertility duration $(31.89 \pm 5.70$ years vs $30.25 \pm 6.80$ years; $\mathrm{p}=0.008$ ), more children (parity $\geq 3$ : $43.3 \%$ vs $18.6 \%$; $<0.001$ ), and longer breast feeding periods ( $\geq 6$ months: 73.2 months vs 55.4 months; $\mathrm{p}<0.001$ ), as well as more frequent postmenopausal status $(84.0 \%$ vs $62.8 \%$; $\mathrm{p}<0.001$ ), compared with patients who had diffusetype GCs. Among the reproductive factors that were significantly associated with intestinal-type GCs in univariate analysis, age at diagnosis of GC (odds ratio [OR], 1.066; $95 \%$ confidence interval [CI], 1.042 to $1.091 ; \mathrm{p}<0.001)$ and parity $\geq 3$ (OR, $1.700 ; 95 \% \mathrm{CI}, 1.055$ to $2.878 ; \mathrm{p}=0.048$ ) remained significantly associated with intestinal-type GC development in multivariate analysis (Table 3).

Separate analyses of postmenopausal and premenopausal females were performed (Tables 2 and 3). Females with intestinal-type GCs were more frequently postmenopausal for $\geq 20$ years, compared with females who had diffusetype GCs $(34.1 \%$ vs $16.3 \%$; $\mathrm{p}<0.001)$. Among postmeno- 


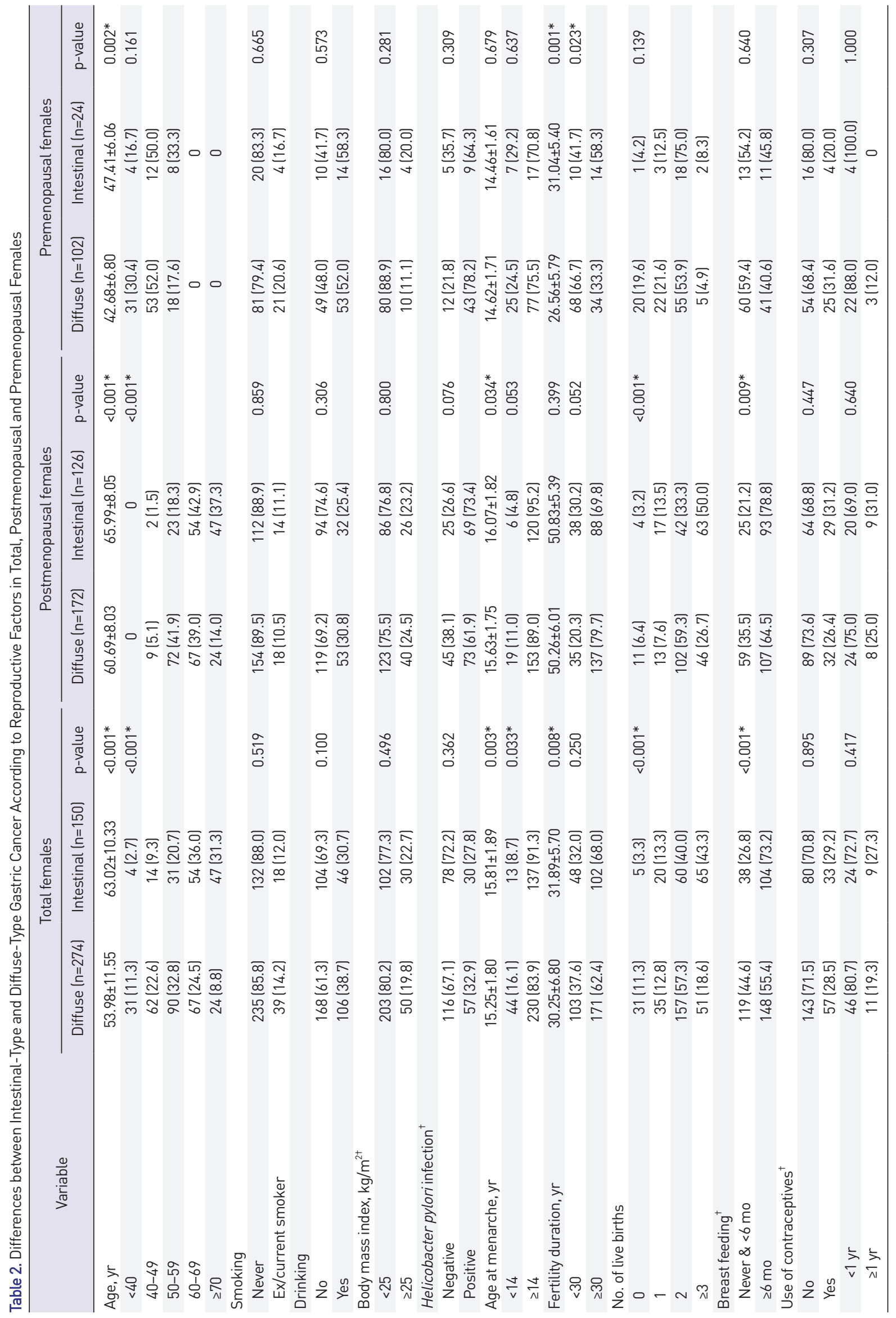




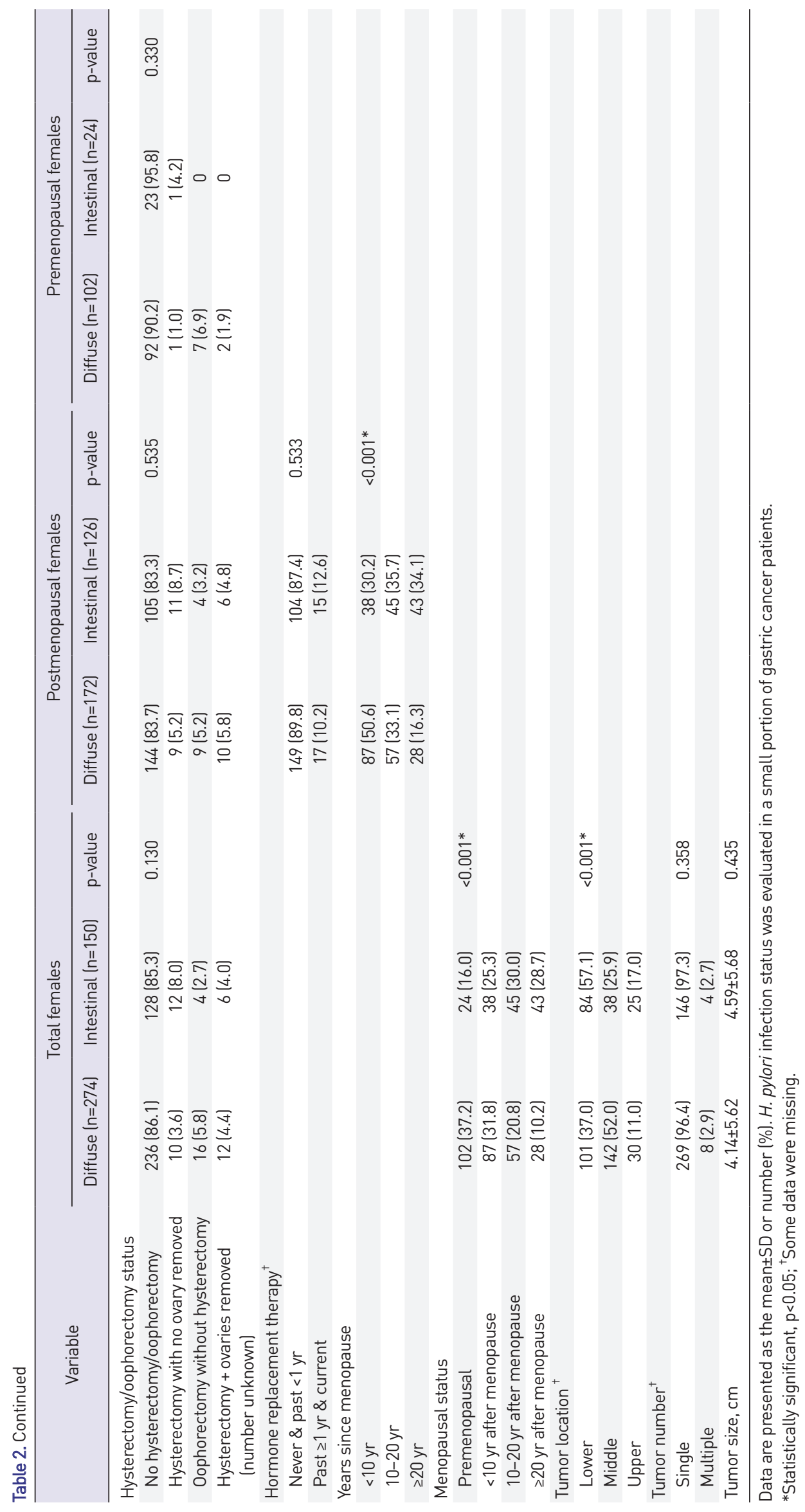


Table 3. Reproductive Factors Associated with Intestinal-Type Gastric Cancer Risk in Multivariate Analysis

\begin{tabular}{lcc}
\multicolumn{1}{c}{ Factor } & Odds ratio (95\% Cl) & $p$-value \\
\hline Total females & & \\
Age at diagnosis of gastric cancer & $1.066(1.042-1.091)$ & $<0.001^{*}$ \\
Age at menarche & $0.974(0.694-1.366)$ & 0.877 \\
Fertility years & $0.962(0.702-1.313)$ & 0.799 \\
Parity $\geq 3$ (vs nulliparity or parity $<3)$ & $1.700(1.055-2.878)$ & $0.048^{*}$ \\
Breast feeding $\geq 6$ mo (no or $<6$ mo) & $1.131(0.665-1.924)$ & 0.651 \\
Menopause (vs premenopausal status) & $0.538(0.252-1.147)$ & 0.109 \\
Postmenopausal females & & $<0.001^{*}$ \\
Age at diagnosis of gastric cancer & $1.075(1.039-1.113)$ & 0.833 \\
Age at menarche & $1.073(0.914-1.260)$ & $0.045^{*}$ \\
Parity $\geq 3$ (vs nulliparity or parity $<3)$ & $1.775(1.012-3.114)$ & 0.672 \\
Breast feeding $\geq 6$ mo (no or $<6$ mo) & $1.144(0.614-2.134)$ & 0.841 \\
$\geq 20$ yr after menopause (vs $<20$ yr after menopause) & $0.908(0.352-2.340)$ & 0.378 \\
Premenopausal females & & $0.005^{*}$ \\
Age at diagnosis & $0.893(0.694-1.148)$ & 0.119 \\
Fertility years & $1.147(1.043-1.261)$ & $2.577(0.783-8.486)$ \\
Parity $\geq 2$ & & \\
\hline
\end{tabular}

$\mathrm{Cl}$, confidence interval.

*Statistically significant, $p<0.05$.

pausal females, age at diagnosis of $\mathrm{GC}(\mathrm{OR}, 1.075 ; 95 \% \mathrm{CI}$, 1.039 to 1.113 ; $\mathrm{p}<0.001$ ), and parity $\geq 3$ (OR, $1.775 ; 95 \% \mathrm{CI}$, 1.012 to $3.114 ; \mathrm{p}=0.045$ ) were factors significantly associated with intestinal-type GCs (Table 3). Among premenopausal females, long fertility duration (OR, 1.147; 95\% CI, 1.043 to $1.261 ; \mathrm{p}=0.005)$ was significantly associated with intestinal-type GCs (Table 3).

\section{DISCUSSION}

In the present study, the proportions of intestinal-type GCs were significantly lower in premenopausal females and $<20$ years postmenopausal females than in males. Females $\geq 20$ years postmenopause had an intestinal-type GC prevalence similar to the prevalence in males. Age and parity were positively associated with an increased risk of intestinal-type GCs in postmenopausal females, while long fertility duration was positively associated with an increased risk of intestinal-type GCs in premenopausal females.

A reported 10- to 15-year delay in the onset of intestinal-type GCs in females suggests that a protective effect is mediated by estrogen. ${ }^{4}$ However, only a few studies have examined relationships between reproductive factors and GC risk based on Lauren subtypes. ${ }^{17-19}$ A case-control study conducted in Canada found that age at menopause, parity ( $>4$ live births), and oral contraceptive use had greater associations with intestinal-type GCs than with diffuse-type GCs. ${ }^{17}$ A large cohort study in Japan found that females with early menarche ( $\leq 12$ years) had an almost $50 \%$ re- duced risk of GCs, compared with females who had late menarche ( $\geq 15$ years); in subgroup analyses according to histologic subtype, lower risk of differentiated-type GC was observed in females with early menarche (13 to 14 years), but no risk reduction was observed for undifferentiatedtype GC. ${ }^{18}$ A recent single center study in Korea found no premenopausal females with intestinal-type GCs; it reported that the incidence of intestinal-type GCs increased over time after menopause, approaching the incidence in males at 10 years postmenopause. That study also revealed an association between parity and an increased risk of intestinal-type GCs in postmenopausal females. ${ }^{19}$ Based on previous studies and our findings, the risk reduction associated with estrogen was significant only for intestinal-type GCs. There have been inconsistent results regarding the relationships between reproductive factors and GC risk in previous studies, which may be related to different proportions of intestinal-type GCs that have been present in those studies.

In the present study, we classified parity as 0,1 to 2 , or $\geq 3$ births to allow analysis over longer periods. We found that $\geq 3$ births were more strongly associated with intestinal-type GCs than with 0 or 1 to 2 births in postmenopausal females, while fertility duration was the only significant factor in premenopausal females. While some previous studies have suggested a positive association between parity and GCs, the results have been inconsistent. Most of the previous studies have not shown any associations with parity or increased number of births. ${ }^{20,21} \mathrm{~A}$ meta-analysis of 10 cohort studies found no significant association between parity and the risk of developing GCs. ${ }^{21}$ In contrast 
to the results of previous studies, we found a significant association between parity and intestinal-type GCs. ${ }^{18,22}$ This may be explained by the effect of childbirth on lifetime exposure to sex hormones. Pregnant females have markedly elevated serum levels of certain hormones, including estrogen, ${ }^{23}$ although a history of more full-term pregnancies is not directly associated with levels of circulating estrogens. Increased parity is associated with an overall increase in exposure to sex hormones. Because the exposure period during the lifespan of a female is insufficient to have an appreciable effect on gastric carcinogenesis, parity might be an inadequate indicator of overall exposure to sex hormones. ${ }^{23}$ Estrogen levels increase markedly during pregnancy, then decrease after childbirth and during lactation. ${ }^{24}$ Parity influences estrogen levels later in life; females with greater parity exhibit lower circulating estrogen levels, compared with females who have lower parity or nulliparity. ${ }^{24}$ Postmenopausal serum-free estradiol decreases with increasing number of childbirths. ${ }^{25}$ Therefore, greater parity might be a risk factor for intestinal-type GCs in postmenopausal females. There have been studies showing an increased number of pregnancies is associated with Alzheimer dementia, consistent with the neuroprotective effects of estrogen in females. ${ }^{26-28}$ Such a discrepancy between postmenopausal and premenopausal females might have been derived from higher prevalence and grade of atrophic gastritis and intestinal metaplasia ${ }^{29}$ with $H$. pylori rates of the aged population compared to young females, ${ }^{30}$ because age and parity $\geq 3$ were risk factors for intestinaltype GC in postmenopausal females. To verify this, data on H. pylori infection status (past, current, or naive) should be added and analyzed. However, it was difficult for getting enough information regarding histologic atrophy and intestinal metaplasia and $H$. pylori infection status in the present study. In addition, only 13 premenopausal females had more than three full-term pregnancies; among these females, only four had intestinal-type GCs. The small number of patients may have affected the results in premenopausal females.

The effects of estrogen on GC development based on Lauren histologic subtypes have not been investigated. Estrogen acts via estrogen receptors (genomic pathway) and transcriptional cross-talk (non-genomic pathway); several studies have investigated the role of estrogen receptors in GC. Wang et al. ${ }^{31}$ reported that well-differentiated gastric adenocarcinomas had higher levels of estrogen receptor- $\beta$ expression, and that poorly differentiated disease was associated with the reduction or loss of estrogen receptor- $\beta$. Yi et al. ${ }^{32}$ demonstrated that estrogen receptor- $\alpha$ expression was associated with diffuse-type GCs and shorter disease-free survival. Several studies have reported that $17-\beta$-estradiol (E2), the most potent isoform of estrogen, downregulated E-cadherin levels via estrogen receptor- $\alpha$ signaling, ${ }^{33-35}$ this downregulation may contribute to the onset of diffuse-type GCs. Further research is necessary to analyze the sex-specific aspects of GCs.

This study had several limitations. First, it used selfreports of reproductive factors, which may be subject to recall bias. Second, this study lacked information regarding H. pylori status in some patients. An increased susceptibility to $H$. pylori infection during pregnancy might be related to increased GC risk. ${ }^{36}$ However, $H$. pylori infection reportedly did not influence the associations of menstrual and reproductive factors with GC risk. ${ }^{37}$ In addition, we did not have information regarding specific types of hormone replacement therapies and oral contraceptives; we also lacked information concerning socioeconomic status, education, and dietary factors. Finally, we did not include patients with unresectable GCs.

In conclusion, we found that age and parity were associated with an increased risk of intestinal-type GCs in postmenopausal females, while longer fertility duration was positively associated with an increased risk of intestinaltype GCs in premenopausal females. The role of estrogen may vary according to GC histology.

\section{CONFLICTS OF INTEREST}

This work was supported by SK Chemical Research Fund of the Korean Society of Gastroenterology. Except for that, no potential conflict of interest relevant to this article was reported.

\section{ACKNOWLEDGEMENTS}

This work was supported by SK Chemical Research Fund of the Korean Society of Gastroenterology.

\section{AUTHOR CONTRIBUTIONS}

Study concept and design: Y.J.J., S.J.P., N.K. Data acquisition: Y.J.J., C.H.P., S.J.P., N.K. Data analysis and interpretation: Y.J.J., H.J.K. Drafting of the manuscript; critical revision of the manuscript for important intellectual content: Y.J.J., H.J.K. Statistical analysis: H.J.K. Obtained funding: Y.J.J. Administrative, technical, or material support; study supervision: S.J.P., N.K. Approval of final manuscript: all authors. 


\section{ORCID}

Yoon Ju Jung

https://orcid.org/0000-0002-3406-1464

Hee Jin Kim

https://orcid.org/0000-0001-9608-0348

Cho Hyun Park

Seun Ja Park

Nayoung Kim

https://orcid.org/0000-0001-5152-4371

https://orcid.org/0000-0003-3217-5115

https://orcid.org/0000-0002-9397-0406

\section{SUPPLEMENTARY MATERIALS}

Supplementary materials can be accessed at https://doi. org/10.5009/gnl210293.

\section{REFERENCES}

1. Global Burden of Disease Cancer Collaboration, Fitzmaurice C, Abate D, et al. Global, regional, and national cancer incidence, mortality, years of life lost, years lived with disability, and disability-adjusted life-years for 29 cancer groups, 1990 to 2017: a systematic analysis for the Global Burden of Disease study. JAMA Oncol 2019;5:1749-1768.

2. Freedman ND, Derakhshan MH, Abnet CC, Schatzkin A, Hollenbeck AR, McColl KE. Male predominance of upper gastrointestinal adenocarcinoma cannot be explained by differences in tobacco smoking in men versus women. Eur J Cancer 2010;46:2473-2478.

3. Ibrahim A, Morais S, Ferro A, Lunet N, Peleteiro B. Sexdifferences in the prevalence of Helicobacter pylori infection in pediatric and adult populations: systematic review and meta-analysis of 244 studies. Dig Liver Dis 2017;49:742-749.

4. Sipponen P, Correa P. Delayed rise in incidence of gastric cancer in females results in unique sex ratio $(M / F)$ pattern: etiologic hypothesis. Gastric Cancer 2002;5:213-219.

5. Wong TS, Chay WY, Tan MH, Chow KY, Lim WY. Reproductive factors, obesity and risk of colorectal cancer in a cohort of Asian women. Cancer Epidemiol 2019;58:33-43.

6. Gruber CJ, Tschugguel W, Schneeberger C, Huber JC. Production and actions of estrogens. N Engl J Med 2002;346:340-352.

7. Camargo MC, Goto Y, Zabaleta J, Morgan DR, Correa P, Rabkin CS. Sex hormones, hormonal interventions, and gastric cancer risk: a meta-analysis. Cancer Epidemiol Biomarkers Prev 2012;21:20-38.

8. Sanikini H, Muller DC, Sophiea M, et al. Anthropometric and reproductive factors and risk of esophageal and gastric cancer by subtype and subsite: results from the European Prospective Investigation into Cancer and Nutrition (EPIC) cohort. Int J Cancer 2020;146:929-942.

9. Lauren $\mathrm{P}$. The two histological main types of gastric carci- noma: diffuse and so-called intestinal-type carcinoma. an attempt at a histo-clinical classification. Acta Pathol Microbiol Scand 1965;64:31-49.

10. Kim KW, Kim N, Choi Y, et al. Different effects of p53 protein overexpression on the survival of gastric cancer patients according to Lauren histologic classification: a retrospective study. Gastric Cancer 2021;24:844-857.

11. Lee JW, Kim N, Kwon YJ, Lee HS. Changes in the clinical and pathological characteristics of gastric cancer during the last 16 years: a study of a single institution in Korea. Korean J Helicobacter Up Gastrointest Res 2019;19:120-126.

12. Correa P. Human gastric carcinogenesis: a multistep and multifactorial process. First American Cancer Society Award lecture on cancer epidemiology and prevention. Cancer Res 1992;52:6735-6740.

13. Exploring the biological contributions to human health: does sex matter? J Womens Health Gend Based Med 2001;10:433439.

14. Chang L, Toner BB, Fukudo S, et al. Gender, age, society, culture, and the patient's perspective in the functional gastrointestinal disorders. Gastroenterology 2006;130:1435-1446.

15. Annandale E, Hammarström A. Constructing the 'genderspecific body': a critical discourse analysis of publications in the field of gender-specific medicine. Health (London) 2011;15:571-587.

16. Kilminster S, Downes J, Gough B, Murdoch-Eaton D, Roberts T. Women in medicine: is there a problem? A literature review of the changing gender composition, structures and occupational cultures in medicine. Med Educ 2007;41:39-49.

17. Frise S, Kreiger N, Gallinger S, Tomlinson G, Cotterchio M. Menstrual and reproductive risk factors and risk for gastric adenocarcinoma in women: findings from the Canadian National Enhanced Cancer Surveillance System. Ann Epidemiol 2006;16:908-916.

18. Persson C, Inoue M, Sasazuki S, et al. Female reproductive factors and the risk of gastric cancer in a large-scale population-based cohort study in Japan (JPHC study). Eur J Cancer Prev 2008;17:345-353.

19. Kim SM, Min BH, Lee J, et al. Protective effects of female reproductive factors on Lauren intestinal-type gastric adenocarcinoma. Yonsei Med J 2018;59:28-34.

20. Endogenous Hormones and Breast Cancer Collaborative Group, Key TJ, Appleby PN, et al. Circulating sex hormones and breast cancer risk factors in postmenopausal women: reanalysis of 13 studies. Br J Cancer 2011;105:709-722.

21. Chen J, Gong TT, Wu QJ. Parity and gastric cancer risk: a systematic review and dose-response meta-analysis of prospective cohort studies. Sci Rep 2016;6:18766.

22. Freedman ND, Chow WH, Gao YT, et al. Menstrual and reproductive factors and gastric cancer risk in a large prospective study of women. Gut 2007;56:1671-1677. 
23. Bahmanyar S, Lambe M, Zendehdel K, Nyrén O, Boffetta $\mathrm{P}, \mathrm{Ye} \mathrm{W}$. Parity and risk of stomach cancer by sub-site: a national Swedish study. Br J Cancer 2008;98:1295-1300.

24. Petrakis NL, Wrensch MR, Ernster VL, et al. Influence of pregnancy and lactation on serum and breast fluid estrogen levels: implications for breast cancer risk. Int J Cancer 1987;40:587-591.

25. Chavez-MacGregor M, van Gils CH, van der Schouw YT, Monninkhof E, van Noord PA, Peeters PH. Lifetime cumulative number of menstrual cycles and serum sex hormone levels in postmenopausal women. Breast Cancer Res Treat 2008;108:101-112.

26. Heys M, Jiang C, Cheng KK, et al. Life long endogenous estrogen exposure and later adulthood cognitive function in a population of naturally postmenopausal women from Southern China: the Guangzhou Biobank Cohort Study. Psychoneuroendocrinology 2011;36:864-873.

27. Prince MJ, Acosta D, Guerra M, et al. Reproductive period, endogenous estrogen exposure and dementia incidence among women in Latin America and China: a 10/66 population-based cohort study. PLoS One 2018;13:e0192889.

28. Jang H, Bae JB, Dardiotis E, et al. Differential effects of completed and incomplete pregnancies on the risk of Alzheimer disease. Neurology 2018;91:e643-e651.

29. Kwon YJ, Kim N, Baek SM, et al. The prevalence of histologic atrophy and intestinal metaplasia in the corpus has decreased over 15 years in females in the Korean population. Helicobacter 2019;24:e12579.

30. Lim SH, Kim N, Kwon JW, et al. Trends in the seroprevalence of Helicobacter pylori infection and its putative eradi- cation rate over 18 years in Korea: a cross-sectional nationwide multicenter study. PLoS One 2018;13:e0204762.

31. Wang M, Pan JY, Song GR, Chen HB, An LJ, Qu SX. Altered expression of estrogen receptor alpha and beta in advanced gastric adenocarcinoma: correlation with prothymosin alpha and clinicopathological parameters. Eur J Surg Oncol 2007; 33:195-201

32. Yi JH, Do IG, Jang J, et al. Anti-tumor efficacy of fulvestrant in estrogen receptor positive gastric cancer. Sci Rep 2014;4: 7592.

33. Park SH, Cheung LW, Wong AS, Leung PC. Estrogen regulates Snail and Slug in the down-regulation of Ecadherin and induces metastatic potential of ovarian cancer cells through estrogen receptor alpha. Mol Endocrinol 2008;22:2085-2098.

34. Helguero LA, Lindberg K, Gardmo C, Schwend T, Gustafsson JA, Haldosén LA. Different roles of estrogen receptors alpha and beta in the regulation of E-cadherin protein levels in a mouse mammary epithelial cell line. Cancer Res 2008;68:8695-8704.

35. Oesterreich S, Deng W, Jiang S, et al. Estrogen-mediated down-regulation of E-cadherin in breast cancer cells. Cancer Res 2003;63:5203-5208.

36. Lanciers S, Despinasse B, Mehta DI, Blecker U. Increased susceptibility to Helicobacter pylori infection in pregnancy. Infect Dis Obstet Gynecol 1999;7:195-198.

37. Freedman ND, Lacey JV Jr, Hollenbeck AR, Leitzmann MF, Schatzkin A, Abnet CC. The association of menstrual and reproductive factors with upper gastrointestinal tract cancers in the NIH-AARP cohort. Cancer 2010;116:1572-1581. 